\title{
Genetic variant in CACNA1C is associated with PTSD in traumatized police officers
}

\author{
Izabela M. Krzyzewska ${ }^{1}$ Judith B.M. Ensink ${ }^{2,3} \cdot$ Laura Nawijn $^{4} \cdot$ Adri N. Mul $\mathbb{D}^{1} \cdot$ Saskia B. Koch $^{4} \cdot$ Andrea Venema $^{1}$. \\ Vinod Shankar ${ }^{1} \cdot$ Jessie L. Frijling ${ }^{4} \cdot$ Dirk J. Veltman ${ }^{5} \cdot$ Ramon J.L. Lindauer $^{2,3} \cdot$ Miranda Olff $^{4,6} \cdot$ \\ Marcel M.A.M. Mannens ${ }^{1} \cdot$ Mirjam van Zuiden $^{4} \cdot$ Peter Henneman $^{1}$
}

Received: 7 March 2017 / Revised: 6 October 2017 / Accepted: 31 October 2017 / Published online: 23 January 2018

(c) The Author(s) 2018. This article is published with open access

\begin{abstract}
Posttraumatic stress disorder (PTSD) is a debilitating psychiatric disorder that may develop after a traumatic event. Here we aimed to identify epigenetic and genetic loci associated with PTSD. We included 73 traumatized police officers with extreme phenotypes regarding symptom severity despite similar trauma history: $n=34$ had PTSD and $n=39$ had minimal PTSD symptoms. Epigenetic and genetic profiles were based on the Illumina HumanMethylation450 BeadChip. We searched for differentially methylated probes (DMPs) and differentially methylated regions (DMRs). For genetic associations we analyzed the CpG-SNPs present on the array. We detected no genome-wide significant DMPs and we did not replicate previously reported DMPs associated with PTSD. However, GSE analysis of the top 100 DMPs showed enrichment of three genes involved in the dopaminergic neurogenesis pathway. Furthermore, we observed a suggestive association of one relatively large DMR between patients and controls, which was located at the PAX8 gene and previously associated with other psychiatric disorders. Finally, we validated five PTSD-associated CpG-SNPs identified with the array using sanger sequencing. We subsequently replicated the association of one common SNP (rs1990322) in the CACNAIC locus with PTSD in an independent cohort of traumatized children. The CACNAIC locus was previously associated with other psychiatric disorders, but not yet with PTSD. Thus, despite the small sample size, inclusion of extreme symptom severity phenotypes in a highly homogenous traumatized cohort enabled detection of epigenetic and genetic loci associated with PTSD. Moreover, here we showed that genetically confounded 450K probes are informative for genetic association analysis.
\end{abstract}

\section{Introduction}

Posttraumatic stress disorder (PTSD) is a debilitating psychiatric disorder that may develop after exposure to traumatic events. The disorder is characterized by recurrent, involuntary re-experiencing of the traumatic event, accompanied by avoidance of trauma-related stimuli; negative alterations in cognition and mood and in reactivity [1]. Its lifetime prevalence in Western countries

Izabela M. Krzyzewska, Judith B.M. Ensink, Laura Nawijn, Mirjam van Zuiden and Peter Henneman contributed equally to this work.

Electronic supplementary material The online version of this article (https://doi.org/10.1038/s41431-017-0059-1) contains supplementary material, which is available to authorized users.

Marcel M. A. M. Mannens

m.a.mannens@amc.uva.nl

Extended author information available on the last page of the article is estimated at $5.7-7.4 \%[2,3]$. In all, the societal impact of PTSD is high, not only because of its negative impact on quality of life of affected individuals and their families, but also due to high economic costs resulting from health care utilization and productivity loss and the high percentage of patients not adequately responding to treatment $[4,5]$. Additionally, PTSD is associated with increased risk for a wide range of severe chronic medical disorders and increased all-cause early mortality [6-8]. Longitudinal studies consistently show that only a subset of individuals will develop PTSD in the aftermath of apparently similar traumatic events, indicating differential susceptibility [9]. To date, the biological mechanisms and etiology underlying differential susceptibility for PTSD remain poorly understood, but accumulating evidence indicates that genetic and epigenetic factors are involved [10, 11].

Genetic factors contribute substantially to PTSD development: its heritability is estimated between 0.28 and 0.46 
[12-14]. PTSD has a highly heterogeneous phenotype and can be characterized as complex trait, indicating that common genetic variation within hundreds to thousands of genes might contribute to its expression. For most of these genetic contributors, small effect sizes can be expected [15]. Moreover, PTSD development cannot be explained by genetic factors alone [12, 16, 17]. PTSD involves by definition exposure to a precipitating traumatic event suggesting an additional role for epigenetic processes that mediate gene regulation under the influence of the environment [18]. Recent insights show that epigenetic associations with psychiatric disorders can not only be detected in the most obvious target tissue, i.e. the brain, but also in peripheral blood [19]. With regard to PTSD, several candidate gene studies have detected genetic and epigenetic associations. However, the number of hypothesis-free, i.e. genome wide genetic and epigenetic studies on PTSD, remains limited [20, 21]. In this study, we aimed to identify epigenetic and genetic loci associated with PTSD in a Dutch cohort of highly trauma exposed police officers with and without PTSD. We selected our cohort to be homogenous, [also] with respect to trauma history, but showing extreme phenotypes regarding PTSD symptom severity (i.e. highly affected vs. resilient with minimal symptoms), maximizing likelihood to detect PTSDrelated associations in a relatively small sample [22]. We used the Infinium HumanMethylation 450 BeadChip of Illumina, a commonly used platform for epigenome-wide association studies. Next to $\sim 430 \mathrm{~K}$ informative epigenetic markers, this array contains a substantial number of probes biased by common genetic variation. These probes involve common single nucleotide polymorphisms at the $\mathrm{CpG}$ site of interest (CpG-SNPs) directly affecting the readout of the methylation array at that position. Usually these probes are discarded from epigenetic surveys, despite the fact that some of the $\sim 14 \mathrm{~K}$ probes show well-defined genotypic clusters that may be used for genetic association analysis. Detailed information on promiscuous $450 \mathrm{~K}$ probes, i.e. genetic confounded and cross hybridizing probes, and the design of the array is described by Chen et al. [23]. In this study, we used these $14 \mathrm{~K}$ CpG-SNP probes for genetic association analyses and subsequently validated identified candidate PTSDassociated SNPs with additional sanger-sequencing. Consistent genetic findings between array and sequencing methods were replicated in a Dutch cohort of trauma exposed children with and without PTSD.

\section{Subjects and methods}

\section{Participants}

This study is part of a larger neuroimaging study on the effects of a single oxytocin administration on neural activity, in which trauma-exposed police officers with and without PTSD between 18 and 65 years old were included [24]. For the current study, blood samples were collected from 73 participants: 34 PTSD patients and 39 controls. PTSD patients fulfilled DSM-IV criteria for current PTSD, with a Clinician Administered PTSD Scale (CAPS) score $\geq$ 45 [25]. Exclusion criteria for PTSD patients were current severe MDD (with psychotic features or suicidal intent), bipolar disorder, suicidal ideation, alcohol/substance abuse, and psychotic disorders, as measured with the Mini International Neuropsychiatric Interview or Structured Clinical Interview for DSM-IV [26, 27]. Controls reported at least one traumatic event (DSM-IV A1 criterion), with a CAPS score $<15$. Controls were matched to PTSD patients based on sex, age, education, and years of service. Exclusion criteria for controls were lifetime MDD or PTSD or any current DSM-IV Axis-I psychiatric disorder. Exclusion criteria for all participants consisted of daily use of psychoactive medication or systemic glucocorticoids, contraindications for MRI or oxytocin administration, serious medical conditions or history of neurological disorders. All participants provided written informed consent prior to study initiation. The study was approved by the Institutional Review Board of the Academic Medical Center, Amsterdam, the Netherlands. Patients were recruited through a diagnostic outpatient center for police personnel with psychological trauma (PDC, Diemen, the Netherlands). Additionally, PTSD patients and controls were recruited through advertisements. Group differences in demographic and clinical characteristics were tested using independent sample $t$-tests (log transformed in case of nonnormally distributed data) for continuous variables and Chisquare tests or Fisher's exact tests for categorical variables, performed in SPSS (version 20.0.0, IBM $^{\circledR}$ SPSS $^{\circledR}$ Statistics).

\section{Procedures}

Sample preparation and quality control of the 450K DNAmethylation array are described in appendix 1 . The $450 \mathrm{~K}$ DNA-methylation array comprises a substantial number of SNPs located in or near $\mathrm{CpG}$ sites at single base extensions (SBE) sites of probes. As was previously shown by others [23], a substantial number of these CpG-SNPs show 3 or 2 well divided clusters of beta-values, reflecting genotypes. Therefore, we divided the probes into two data sets, one for epigenetic and one for genetic association analysis (see Figure S1 for schematic overview). For both data sets promiscuous $450 \mathrm{~K}$ probes, i.e. cross hybridizing probes, were excluded from analysis [23]. In addition, probes located on both the $\mathrm{X}$ and $\mathrm{Y}$ chromosomes were not included in further analysis. The epigenetic probe set included 432.222 probes, excluding probes with a common 
SNP with a CEU minor allele frequency (maf) $>0.01$ located at the SBE site. This probe set was used to identify differentially methylated probes (DMPs) and differentially methylated regions (DMRs). The CpG-SNP genetic probe set included the 13.344 probes with a common SNP located at SBE site with a CEU maf $>0.01$. Unprocessed genome wide epigenetic and meta data are freely available NCBI Gene Expression Omnibus (GEO, https://www.ncbi.nlm. nih.gov/geo) Accession ID: GSE99755

\section{Epigenetic association analyses}

A complete description of DMP and DMR analyses is provided in appendix 1. For both DMP and DMR analyses, we included the following covariates in the model: age, leukocyte cell distribution (CD8+ and CD4+ T cells, Natural killer cells, B cells, Monocytes, and Granulocytes), and sex. For DMP association analyses $\operatorname{lm}$ fit ("R"package limma) function was used. We assumed a $q$-value $<0.05$ (Benjamini-Hochberg adjusted alpha) as genome-wide significant. In order to replicate previously reported epigenetic associations (DMPs) with PTSD we additionally applied a hypothesis-driven approach; we performed a NCBI PubMed search using keywords "450K", "Illumina" and "PTSD". Heterogeneity between studies complicates interpretation and power of the replication analyses. We therefore applied strict inclusion criteria on PTSD characterization, technical platform, abuse history, and mean age of the sample and the type of tissue of which DNA was obtained. From the identified studies we selected the candidate DMPs that were previously reported to be genomewide significantly associated with PTSD, and for these DMPs data was extracted from our association data. Subsequently, we performed gene-set enrichment (GSE) analyses on our top 100 DMP hits, excluding DMPs located at intergenic regions (IGRs, $>1 \mathrm{~kb}$ distance from the gene, 26 DMPs removed, 74 DMPs remained). Enrichment analyses were done via the publicly available consensus pathway database web tool, developed by the Max Planck institute, version 31, 2015 [28].

DMR association analyses were performed using the minfi function bumphunter. DMRs were defined as including two or more probes $(L>1)$ in the bump. Genome wide significant DMRs (adjusted $P$-value $<0.05$ according the familywise error-rate over the area of the bump, FWER) or DMRs showing consistent differential methylation over more than $75 \%$ of the probes within the cluster were considered promising. Technical validation was performed targeting one promising DMR (PAX8) and one randomly chosen region located at the SOSTDCl gene. The latter region was included to achieve a broader range of $\beta$-values that favors proper correlation analysis between the two methods. SOSTDCl expressed average $\beta$-values
$<50 \%$ within that region. Technical validation was performed through targeted amplicon sequence analysis using the Illumina MiSeq platform (details see appendix 1).

\section{Genetic association analyses}

In order to detect genetic associations between PTSD and the $450 \mathrm{~K}$ CpG-SNPs, we performed association analyses that conform the above described statistical methodology (epigenetic association analyses). We included only CpGSNPs located at the SBE site or subsequent guanine base with a maf $<0.01$. Secondly, the dataset was filtered on CpG-SNPs showing a delta mean beta difference exceeding $15 \%$ and a nominal $P$-value $<0.05$, which yielded $15 \mathrm{CpG}$ SNPs showing well divided clusters of beta-values (representing the genotypes). Since our study is limited in statistical power, only five CpG-SNPs, showing the lowest nominal $P$-value and most well divided clusters, were followed up by a technical validation using sanger sequencing. Validation (and replication, described below) $P$-values $<$ 0.01 were assumed significant according a Bonferroni correction, based on five independent tests. Since the amount of collected DNA was limited, we first applied a whole genome amplification for all available samples using the Qiagen REPLI-g Mini Kit ${ }^{\mathrm{TM}}$ conform the manufacturers protocol, yielding $>4 \mu \mathrm{g}$ of DNA for each participant. Primer sets for candidate SNPs were designed using Primer3 [29], all primers were M13 extended for standardized Sanger sequencing (Table S1). For validation association analyses genotypes were recoded into ordinal variables followed by Chi-square tests assessing genotype differences between PTSD patients and controls, performed in SPSS (version 20.0.0, IBM ${ }^{\circledR}$ SPSS $^{\circledR}$ Statistics). $P$-values at which significance was assumed were Bonferroni corrected for the number of tests performed $(0.05 /$ number of tests).

Replication analyses of validated CpG-SNPs were performed in a cohort of trauma-exposed children with $(N=$ 46) and without PTSD $(N=50)$, see appendix 1 for details on participant characteristics and genotyping procedure. Epigenetic profiles may differ in a greater or lesser extent between tissues. Since DNA of replication cohort was extracted from saliva and DNA of the discovery cohort was obtained from whole blood we considered only replication of genetic associations in the discovery cohort appropriate. Statistical analyses were performed as described above for validation analyses. $P$-values at which significance was assumed were Bonferonni corrected for the number of tests performed (0.05/number of tests). Genotypes and metadata of the five reported SNPs for all individuals of the replication cohort are available in Table S3-tab Genotypes_Replicationcohort 


\section{Results}

\section{Discovery cohort participant characteristics}

PTSD patients and trauma-exposed controls did not differ on demographic or trauma history characteristics. As expected, PTSD patients had significantly higher PTSD symptom severity and more co-morbid major depressive disorder (MDD) than controls (Table 1).

\section{Sample preparation and quality control $450 \mathrm{~K}$ array}

Quantile normalization yielded proper data normalization (Figure S2). Table S2 lists the estimated relative blood cell count for which we did not observe significant group differences. Principal components (PC) one and two explained most of the variance in the $450 \mathrm{~K}$ data set, 10.1 and $4.1 \%$, respectively (Figure S3A). Pearson's correlation analyses were performed between the first eight PCs with potential covariates/confounders. As several blood cell count estimates showed small to moderate correlations ( $r$ 's $0.2-0.5$ ) with PCs 1 and 2, all blood cell estimates were included as covariates in further analyses. Additionally age and sex were included as covariates in further epigenetic association analyses, although these showed low correlations with PCs 1 and 2 (Figure S3B/C).

\section{Epigenetic association analyses}

We observed no significant global DNA-methylation group difference. Furthermore, we found no genome-wide significant PTSD-associated DMPs (Table S3-tab DMPs, and Figure S4). Subsequently, we addressed a hypothesis-driven approach, evaluating previously reported PTSD associated genome-wide DMPs in our cohort. Our literature search yielded two genome-wide epigenetic surveys for PTSD, both using Illumina $27 \mathrm{~K}$ arrays. Uddin et al. reported only on PTSD-associated regions or nearby genes, while Smith et al. reported on individual PTSD-associated DMPs [20, 21]. Therefore, only the latter was considered appropriate for DMP replication. None of the 21 significant loci reported by Smith et al. reached significance in our study ( $P$-values $<2.4 \mathrm{E}-3$ were assumed significant, Bonferronicorrected). Nevertheless, for these 21 DMPs we did observe an overlap of $94.7 \%$ with regard to the direction of effect, i.e. hypo or hyper methylation in PTSD cases. Finally, we performed GSE analyses on the top 100 DMPs. DMPs located within the vicinity of $1 \mathrm{~kb}$ of a gene, annotated

Table 1 Demographic, trauma history and clinical characteristics of discovery cohort

\begin{tabular}{|c|c|c|c|}
\hline & $\begin{array}{l}\text { PTSD patients }(n= \\
34)\end{array}$ & $\begin{array}{l}\text { Traumatized controls }(n= \\
\text { 39) }\end{array}$ & $P$-value \\
\hline Age & $41.45(8.96)$ & $40.15(10.12)$ & $0.569^{\mathrm{a}}$ \\
\hline Gender (females) & $16(47.1 \%)$ & $19(48.7 \%)$ & $0.887^{\mathrm{b}}$ \\
\hline Years of service & $17.74(13.43)$ & $18.60(9.93)$ & $0.377^{\mathrm{c}}$ \\
\hline Educational level & & & $0.745^{\mathrm{d}}$ \\
\hline Low & $0(0.0 \%)$ & $0(0.0 \%)$ & \\
\hline Middle & $29(87.9 \%)$ & $33(84.6 \%)$ & \\
\hline High & $4(12.1 \%)$ & $6(15.4 \%)$ & \\
\hline Current smoker & $7(21.9 \%)$ & $8(20.5 \%)$ & $0.889^{\mathrm{b}}$ \\
\hline $\begin{array}{l}\text { PTSD symptom severity (CAPS total } \\
\text { score) }\end{array}$ & $70.21(13.43)$ & $4.59(4.73)$ & $<0.001^{\mathrm{a}}$ \\
\hline $\begin{array}{l}\text { Current co-morbid major depressive } \\
\text { disorder }\end{array}$ & $10(29.4 \%)$ & $0(0.0 \%)$ & $<0.001^{\mathrm{d}}$ \\
\hline $\begin{array}{l}\text { Work-related traumatic events (PLES } \\
\text { total score) }\end{array}$ & $19.13(8.65)$ & $19.74(6.78)$ & $0.740^{\mathrm{a}}$ \\
\hline $\begin{array}{l}\text { Childhood traumatic events (ETI total } \\
\text { score) }\end{array}$ & 5.64 (469) & $3.95(3.80)$ & $0.116^{\mathrm{c}}$ \\
\hline
\end{tabular}

Continuous variables presented as mean (standard deviation); categorical variables presented as frequency

(percentage)

PTSD post-traumatic stress disorder, $M D D$ major depressive disorder, CAPS clinician administered PTSD scale, PLES Police LifeEvent Scale, ETI Early Trauma Inventory

${ }^{\mathrm{a}}$ Independent $t$-test

${ }^{\mathrm{b}}$ Fisher's exact test

${ }^{\mathrm{c}}$ Independent $t$-test on log-transformed data

${ }^{\mathrm{d}}$ Chi-square test 
according to the Illumina manifest, were included. We observed 12 enriched pathway-based sets (49 of 74 genes entered GSE) of which six reached significance according to an adjusted $P$-value $(q)<0.05$. The lowest $P$-value we detected involved the dopaminergic neurogenesis pathway ( $q=0.012$-wikipathways database), including three DMPs located at PITX3, NEUROD1, and the RET genes (Table S3-tabs top100 DMPs/GSE analyses and Figure S6). A secondary GSE analysis was based on the fifth category of the Gene Ontology (GO) database, which yielded only two significant enriched GO terms, both related to molecular functions involved in DNA binding (Table S3—tab top100 DMPs/GSE analyses).

There were no DMRs between PTSD patients and controls that reached genome-wide significance, however for one DMR we did observe consistent hyper methylation over more than $75 \%$ of the probes within the cluster (Table 2), located at the $P A X 8$ gene (Fig. 1a). Technical validation of $450 \mathrm{~K}$ data, i.e. including PAX8 (Fig. 1a and SOSTDC1 Fig. 1b) using MiSEQ technology was successful $\left(r^{2}=0.85\right.$, Fig. 1c) and described in detail in appendix 1.

\section{Genetic association analyses}

For the genetic survey we analyzed 13.344 CpG-SNP probes (maf $>0.01$ ), which yielded $15 \mathrm{CpG-SNPs}$ potentially associated with PTSD. Since the statistical power in this study is limited due to the small sample, only the top 5 CpG-SNPs were investigated further (Figure S5). These five candidate CpG-SNPs were located in or nearby the $O R 2 \mathrm{~V} 2$, EIF3B, SDK1, SKA2, and CACNAIC genes (Table 3, column discovery $P$-value). Next we aimed to validate these five candidate CpG-SNPs using sanger sequencing (Table S4). We did not detect any genotype discrepancies between $450 \mathrm{~K}$-based and sanger-sequencing-based genotypes. Three of five candidates, located at the genes $S D K 1$, $S K A 2$, and $C A C N A 1 C$, were significantly associated with PTSD in this validation (Table 3 , column validation $P$ value). Next we performed replication analysis for the five candidate CpG-SNPs in an independent cohort of trauma- exposed children with and without PTSD (see appendix 1 and Table S5). We significantly replicated the association of one CpG-SNP (rs1990322) located at the CACNA1C locus with PTSD (Table 3 , column replication $P$-value. In addition, for this replicated $\mathrm{CpG-SNP}$ at the CACNAIC locus we searched for evidence for genetic interaction with flanking $450 \mathrm{~K}$ probes (cis meQTLs) within a window of $500 \mathrm{bp}$ upstream and $500 \mathrm{bp}$ downstream in the discovery dataset. We observed no indications for meQTL effects of rs1990322 and its neighboring CpG sites (Figure S7).

\section{Discussion}

In this study we aimed to detect epigenetic and genetic loci associated with PTSD in a homogeneous cohort of traumatized police officers. Both a genome-wide and hypothesis-driven replication approach did not result in DMPs between PTSD patients and trauma-exposed controls. GSE analysis on the top 100 DMPs showed, however, a plausible association of the dopaminergic neurogenesis pathway with PTSD. Furthermore, we observed one DMR located at the PAX8 gene suggesting consistent hypermethylation in PTSD patients. Genetic analyses yielded three CpG-SNPs significantly associated with PTSD. Of these, one CpG-SNP, located at the CACNAIC locus, was also significantly associated with PTSD in an independent replication sample of trauma-exposed children. Notably, this result shows that the Illumina $450 \mathrm{~K}$ array is not restricted to epigenetic surveys but can provide informative genetic data as well.

Although our sample was small, it was highly homogenous as all participants were former or current police officers, and cases and controls were matched for sex, age, education, and years of police service. All participants reported multiple prior traumatic events, without significant group differences in reported types of traumatic experiences. PTSD patients fulfilled current diagnostic criteria for PTSD, while our trauma-exposed controls had minimal PTSD symptoms and did not report lifetime PTSD or other

Table 2 DMR association analysis PTSD vs. traumatized controls

\begin{tabular}{|c|c|c|c|c|c|c|c|}
\hline Gene & chr: start-end & Value & Area & FWER & $L$ & Cluster $(L)$ & $\% L$ \\
\hline PAX8 & chr2:113992762-113993142 & 0.07 & 0.42 & 0.82 & 6 & 8 & 0.75 \\
\hline LDHC & chr11:18433500-18433564 & 0.07 & 0.20 & 0.98 & 3 & 9 & 0.33 \\
\hline FAM71F1 & chr7:128354862-128354967 & -0.07 & 0.20 & 0.98 & 3 & 7 & 0.43 \\
\hline
\end{tabular}

Detected DMRs $(L>2)$ using minfi's "bumphunter" function in whole sample, female and male stratified analyses. chr: chromosome and position (hg19); Value: average difference in methylation in the bump with respect to controls, $<0$ represents hypo methylation in PTSD and $>0$ represents hypermethylation in PTSD; Area: area of the bump with respect to the 0 line; FWER: multiple test adjusted $P$-value with respect to the area, according the familywise error rate; $L$ : number of probes in DMR; cluster $(L)$ : number of probes in cluster; \% $\%$ : percentage of probes within the cluster contributing to the DMR-in bold $\geq 75 \%$ 
Fig. 1 Technical validation of $450 \mathrm{~K}$ data. a DMR PAX8 using the bsr amplicon MiSEQ method and b $450 \mathrm{~K}$ data SOSTDC1 using the bsr amplicon MiSEQ (lower plot) method. For both a and $\mathbf{b} X$-axis plots represents chromosomal position (hg19),

$Y$-axis plots represents $\beta$-values of $450 \mathrm{~K}$ data and MiSEQ data, respectively. Pink line represents PTSD cases and blue line represents traumatized controls. Shading represent $95 \%$ C.I. c Overall correlation plot of $450 \mathrm{~K}$ and MiSEQ detected $\mathrm{CpG}$ sites
(A) Technical validation of 450K data of DMR PAX8 using the bsr amplicon MiSEQ method.

Region chr2: $113992762-113993142$

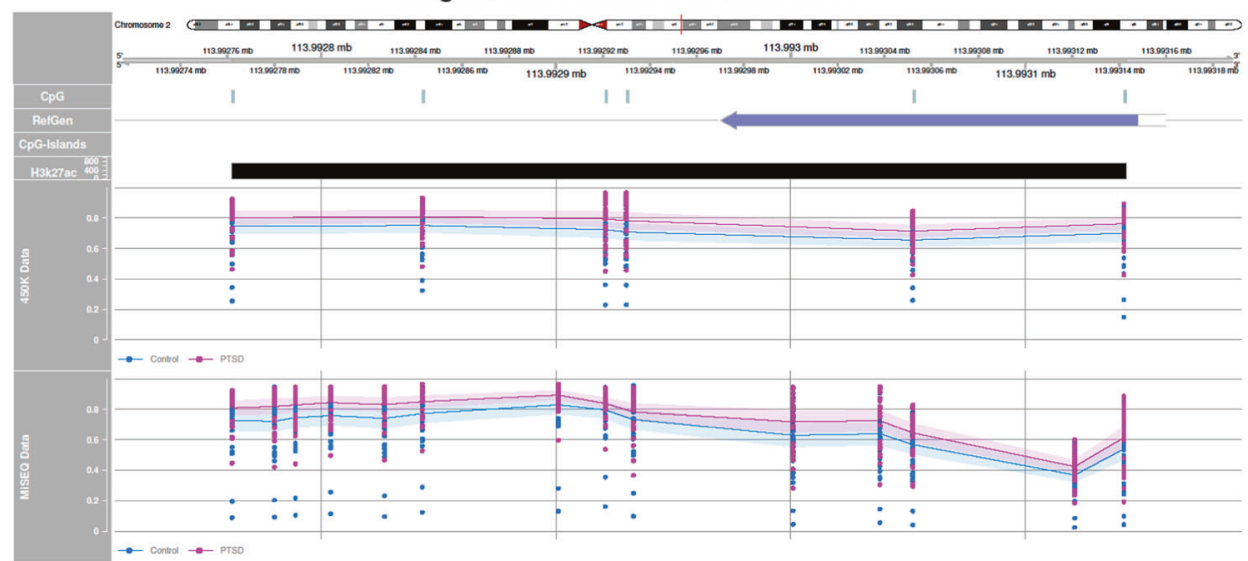

(B) Technical validation of 450K data of SOSTDC1 using the bsr amplicon MiSEQ method.

Region chr7: 16505409-16505664

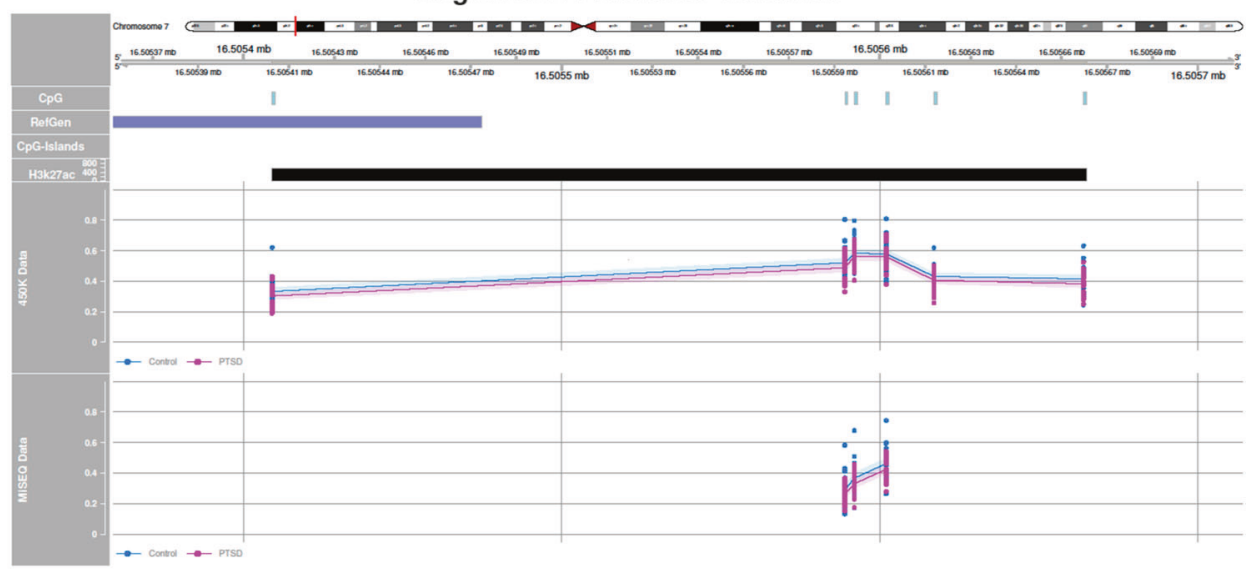

(C) Overall correlation between $450 \mathrm{~K}$ and MiSEQ.

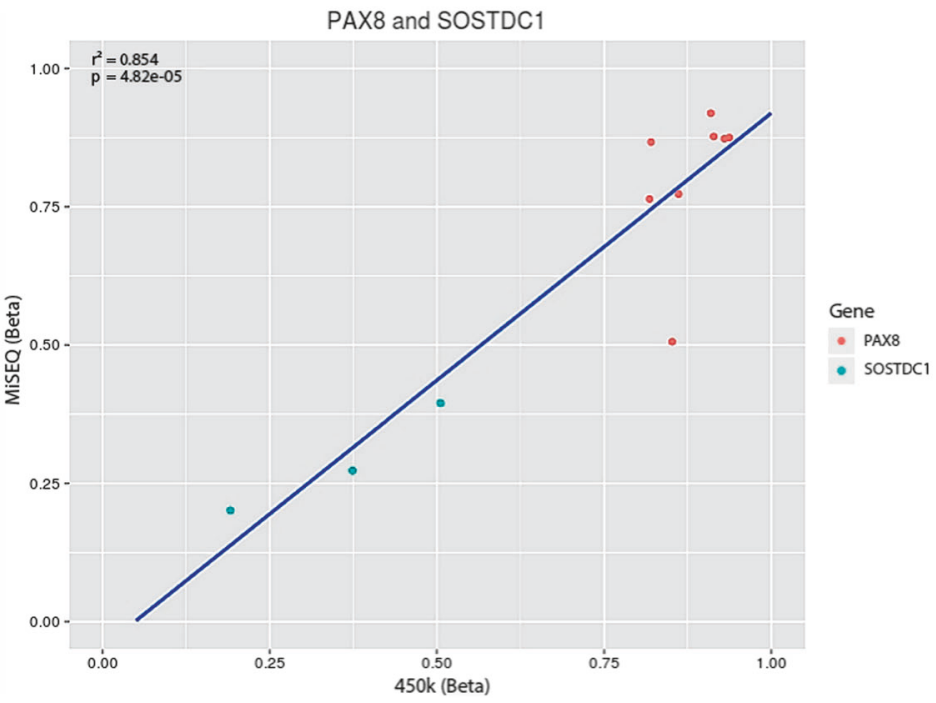


Table 3 Top 5 CpG-SNPs genetic association analysis PTSD vs. traumatized controls

\begin{tabular}{|c|c|c|c|c|c|c|}
\hline rs ID & $\mathrm{HGVS}^{\mathrm{a}}$ (chr) & Probe ID & Gene & $\begin{array}{l}\text { Discovery } \\
\left(P \text {-value }^{\mathrm{b}}\right)\end{array}$ & $\begin{array}{l}\text { Validation } \\
\left.(P \text {-value })^{c}\right)\end{array}$ & $\begin{array}{l}\text { Replication } \\
(P \text {-value })\end{array}$ \\
\hline rs2546424 & $\begin{array}{l}\text { g. } 180581761 \mathrm{C}>\mathrm{T} \\
\text { (5) }\end{array}$ & $\operatorname{cg} 22931151$ & OR $2 \mathrm{~V} 2$ & 0.0009 & $>0.01$ & $>0.01$ \\
\hline rs7808948 & g. $2425741 \mathrm{C}>\mathrm{T}$ (7) & $\operatorname{cg} 03122926$ & $E I F 3 B^{\mathrm{d}}$ & 0.0040 & $>0.01$ & $>0.01$ \\
\hline rs 13230345 & $\begin{array}{l}\text { g. } 4118584 \mathrm{G}>A \\
\text { (7) }\end{array}$ & $\operatorname{cg} 22535849$ & $S D K 1$ & 0.0027 & 0.005 & $>0.01$ \\
\hline rs7208505 & $\begin{array}{l}\text { g. } 57187729 \mathrm{G}>A \\
\text { (17) }\end{array}$ & $\operatorname{cg} 13989295$ & SKA2 & 0.0020 & 0.009 & $>0.01$ \\
\hline rs1990322 & $\begin{array}{l}\text { g. } 2760970 \mathrm{G}>A \\
\text { (12) }\end{array}$ & $\operatorname{cg} 24393317$ & CACNA1C & 0.0006 & 0.006 & 0.001 \\
\hline
\end{tabular}

chr chromosome

${ }^{a}$ Human Genome Variation Society nomenclature (hg19), the probe position and position of the SNP might show a difference of one bp since some of our reported variants are exactly located on the cytosine of the $\mathrm{CpG}$ while other variants are located on the guanine of the CpG which results in exactly the same methylation pattern

${ }^{\mathrm{b}}$ Detection $P$-value-based linear model association

${ }^{c}$ Validation and replication $P$-value-based on Chi-square tests, replication $P<0.01$ was assumed significant (Bonferroni, five tests)

${ }^{\mathrm{d}}$ The rs7808948 is located in the intergenic region between the EIF3B and CHST12 genes

trauma-related psychiatric disorders. Thus our controls were apparently resilient to adverse mental health outcome of trauma. This study design, including extreme phenotypes following similar trauma load, was considered to favor detection of PTSD-associated loci, as also suggested by others [22]. Nevertheless, our genome-wide survey clearly remains limited in statistical power. Post hoc evaluation of the statistical power of our genome wide epigenetic survey was based on the report of Tsai and Bell [30]. If we would assume that our effect sizes ranged between 5 and $10 \%$ and we would aim for a $80 \%$ power, we would need approximately a sample size of 100 per group. The absence of genome-wide significant DMPs is therefore not unexpected. With regard to replication of literature reports on genome wide significant DMPs associated with PTSD, our search resulted in one previous study [20] reporting genome-wide detected DMPs, albeit in a different ethnic group and with substantial lower probe density of the array (27K) [20]. Such methodological differences may explain why we were unable to replicate these DMPs although we did observe a $94.7 \%$ consistent direction of effect between our study and the study of Smith et al.

GSE analysis using the top 100 DMPs revealed three genes, PITX3, NEUROD1, and RET, annotated in the pathway of dopaminergic neurogenesis pathway (wikipathways), which could very plausibly be involved in PTSD. Considering its role in stress reactivity and anhedonia, the dopamine system has previously been implicated in PTSD, and dopamine dysfunctions have been observed in PTSD patients [31]. Interestingly, these genes are involved in neuron differentiation and/or maturation but not neuron regionalization and specification. Differentiation and maturation processes might be more susceptible to adaptation under the influence of the environment, which is clearly relevant for PTSD. Further translational research should be performed in order to unravel the role of this pathway in PTSD.

Compared to DMP analyses, DMR analyses suffer less from statistical power issues, since these follow a multiprobe association methodology implying a reduced multiple test penalty [32]. We detected no DMRs with genome-wide significance after family-wise error rate correction. Such corrections however may be considered too conservative in genome wide screens, given that no false positives are allowed [33]. We did observe one suggestive associated DMR in the PAX8 gene that we consider a promising candidate for further studies on PTSD, since it involved $>75 \%$ concordant differential hypermethylated probes within the cluster window in the PTSD patients. The PAX8 gene encodes a transcription factor involved in thyroid and central nervous system (CNS) development. Interestingly, thyroid hormone alterations have been observed in PTSD patients [34]. Additionally, PAX8 has been repeatedly implicated in sleep disturbances, which constitute an important symptom of PTSD [35]. Furthermore, Siegmund et al. reported hypomethylation of $P A X 8$ in schizophrenia patients, suggesting a broader role for this gene in psychiatric disorders [36].

To our best knowledge, we are the first to use the $\sim 14 \mathrm{~K}$ CpG-SNPs with maf $>0.01$, present on the $450 \mathrm{~K}$ DNAmethylation array of Illumina for genetic association analyses. Although this CpG-SNP set is far too small for 
imputation and for a substantial number of these CpG-SNP genotypes cannot be reliably scored due to interfering DNA methylation effects, we were still able to detect five CpGSNPs potentially associated with PTSD, of which three (SDK1, SKA2, and CACNA1C) were significantly associated with PTSD in the validation analyses. A statistical power estimation (www.osse.bii.a-star.edu.sg) based on the sample size of our discovery cohort showed that in order to achieve a power $\geq 80 \%$ at an alpha of $5 \%$, we should observe a maf difference between cases and controls of $\sim 30 \%$. Since we have observed maximum maf differences of $22 \%$ (for the CACNAIC and SKA2 annotated CpGSNPs) between cases and controls, this indicated a limited statistical power and thus the importance of replication.

Importantly, one of these CpG-SNPs (rs1990322, cg24393317, CACNA1C) was also significantly associated with PTSD in an independent cohort of traumatized children with and without PTSD. This locus is located in an intronic region of the CACNAIC gene. This gene encodes for an alpha-1C subunit of the L-type voltage-dependent calcium channel, crucial for generating neuronal action potentials. Several genome-wide and candidate-gene studies have reported associations between this gene and susceptibility for psychiatric disorders, including MDD [37], and bipolar disorder and schizophrenia [38]. Additionally, CACNA1C SNPs repeatedly have been found to affect brain structure and function, including amygdala responsivity, commonly considered a biological hallmark of PTSD [39]. However, in a candidate-gene study, another common SNP at CACNAIC locus did not moderate between hurricane exposure and subsequent PTSD in Hispanic Blacks [40].

Although our PTSD-associated SNP (rs 1990322) is located in the CACNAIC gene itself, we cannot rule out a long-range interaction of this SNP with the upstream neighboring gene $F K B 4$. FKBP4 is a functional antagonist of $F K B P 5$, modulating glucocorticoid receptor function. Although psychiatric studies of FKBP4 are limited, FKBP5 SNPs, methylation and gene expression have been implicated in several stress-related psychiatric disorders including PTSD [18], and pre-trauma FKBP5 gene expression was found to predict PTSD symptom development upon military deployment [41]. Further studies should elucidate the actual affected gene contributing to the expression of PTSD.

Although the association between PTSD and the SKA2 gene (cg13989295, rs 7208505) in the discovery cohort was only technically validated but not replicated, this observation supports the accumulating evidence for the role of this gene in PTSD and PTSD-related clinical and biological phenotypes. SKA2 modulates glucocorticoid receptor function [42], which was previously found to predict PTSD symptom development after military deployment [41]. Within that same cohort it was recently observed that decreasing SKA2 methylation was associated with PTSD symptom development during deployment, depending on the exact genotype carried [43]. In addition, in another military cohort, genotype carrier status and genotypeadjusted methylation status of this locus was also associated with PTSD [44]. In highly trauma-exposed civilians, similar associations emerged for this locus after controlling for childhood trauma [43]. SKA2 genotype status, genotypeunadjusted and adjusted methylation, and gene and protein expression have also been associated with suicide risk, either directly or in interaction with trauma-exposure or anxiety level $[45,46]$. As several studies reported differential SKA2-methylation between (prospective) psychiatric patients and controls when adjusting for genotype, we additionally investigated this in the discovery cohort conform methods described by Guintivano et al. [43, 46]. We found no evidence for epigenetic effects additional to the CpG-SNP association (data not shown). This may be explained by the fact that epigenetic effects at the $S K A 2$ locus can only occur in $\mathrm{C}$ allele carriers and our sample includes a limited number of homozygous SKA2 C allele carriers, limiting statistical power to detect such an epigenetic effect.

We also observed a technical valid, but not replicated association between PTSD and CpG-SNP rs13230345 (cg22535849). This SNP is located in an intronic region (c.3203-511) of the SDK1 gene [47], encoding for the sidekick cell adhesion molecule 1 . In silico evaluation of this variant showed that it might introduce a novel donor and acceptor splice site (Alamut Visual version 2.7, Interactive Biosoftware, Rouen, France), which in turn might affect transcription, leading to the absence or a dysfunctional protein. Interestingly, the SDKI gene has been implicated in schizophrenia [48] and autism [49], and altered SDK1 methylation was observed in post-mortem brain tissue of MDD patients [47]. Furthermore, Bagot et al. investigated transcriptional regulation of stresssusceptibility in rats and found that $S D K 1$ was associated with social interaction and anxiety behaviors [50].

It should be noted that despite our careful considerations with regard to the phenotypic character, i.e. PTSD cases and resilient controls, defining both our discovery and replication cohort, differences related to the mean age between these two cohorts might be involved in the absence of replication of the genetic variants in SKA2 and SDK1. While our controls in the discovery cohort typically reported multiple prior traumatic experiences (including various types of potentially traumatic experiences during childhood), the majority of controls in the replication sample reported a single traumatic experience. As PTSD risk increases dose-dependently with increasing number of traumatic experiences, it could be that the non-replicated SNPs are involved in PTSD susceptibility in the context of 
increasing trauma load. Such an interaction between SNPs and increasing trauma load was previously observed by Kolassa et al. [51] Such interaction would also explain why previously reported findings on $S K A 2$ and $S D K 1$ in relation to PTSD or related psychological traits in adults, is in line with our observations in our (adult) discovery cohort.

In summary, while our statistical power to detect genome-wide significant DMPs was low, we detected an enriched gene set in the dopaminergic neurogenesis pathway that plausibly plays a role in PTSD. In addition, we observed a suggestive association of one DMR located at the PAX8 gene that seems a good candidate for future studies in PTSD. Furthermore, genetic association analyses based on 450k methylation data, and subsequent validation and replication analyses revealed that the CpG-SNP located at the CACNAIC locus was consistently associated with PTSD both in our discovery cohort and replication cohort. Despite the fact that we were unable to replicate the CpGSNPs located at $S D K 1$ and $S K A 2$ loci, previously reported findings of others and our data do suggest a role of these genes in PTSD.

Additional research has to be performed to elucidate whether the identified DMRs and SNPs are consistently and causally associated with development or expression of PTSD, and importantly with which specific symptoms and biological mechanisms they are associated. For this purpose, transdiagnostic approaches among different types of psychiatric disorders seem especially fruitful.

If replicated, these findings may eventually contribute to the development of PTSD biomarker sets that can potentially support or improve an early clinical diagnosis of PTSD or aid in identifying individuals at risk for PTSD.

\section{Web Resources}

Consensus pathway database web tool, developed by the Max Planck institute, www.consensuspathdb.org, version 31, 2015 [28].

\section{Accession Numbers}

Raw and processed data are freely available on reasonable request but under the strict condition that meta-data remains confidential. Summary statistics on DMPs are given in Table S3.

Acknowledgements The authors would like to thank all participants for their contribution to this study. We additionally would like to thank Renée Hutter, Gré Westerveld, Marthe Hoofwijk, and colleagues of the PDC police outpatient clinic and Bart Timmermans, Els van Meijel, Maj Gigengack, Jasper Zantvoord, and Rosanne op den Kelder for their assistance in participant recruitment and data collection; and
Peter Boorsma and Karin van der Lip from the Department of Clinical Genetics, Genome Diagnostics Laboratory, Amsterdam Medical Center, Amsterdam, The Netherlands for their technical assistance. This research was supported by grants from ZonMw, the Netherlands organization for Health Research and Development (grant no. 91210041, discovery cohort), the Academic Medical Center Research Council (grant no. 110614, discovery cohort), and Augeo Foundation, Driebergen-Rijsenburg, The Netherlands, a Foundation dedicated to the prevention of adverse childhood experiences (genetic and epigenetic analyses).

\section{Compliance with ethical standards}

Conflict of interest The authors declare that they have no conflict of interest.

Open Access This article is licensed under a Creative Commons Attribution-NonCommercial-NoDerivatives 4.0 International License, which permits any non-commercial use, sharing, distribution and reproduction in any medium or format, as long as you give appropriate credit to the original author(s) and the source, and provide a link to the Creative Commons license. You do not have permission under this license to share adapted material derived from this article or parts of it. The images or other third party material in this article are included in the article's Creative Commons license, unless indicated otherwise in a credit line to the material. If material is not included in the article's Creative Commons license and your intended use is not permitted by statutory regulation or exceeds the permitted use, you will need to obtain permission directly from the copyright holder. To view a copy of this license, visit http://creativecommons.org/licenses/by-nc-nd/4.0/.

\section{References}

1. Diagnostic and statistical manual of mental disorders, 5th ed. Proceedings of the American Psychiatric Association. Washington, DC: American Psychiatric Association; 2013.

2. de Vries GJ, Olff M. The lifetime prevalence of traumatic events and posttraumatic stress disorder in the Netherlands. J Trauma Stress. 2009;22:259-67.

3. Kessler RC, Petukhova M, Sampson NA, Zaslavsky AM, Wittchen HU. Twelve-month and lifetime prevalence and lifetime morbid risk of anxiety and mood disorders in the United States. Int J Methods Psychiatr Res. 2012;21:169-84.

4. Pietrzak RH, Goldstein RB, Southwick SM, Grant BF. Physical health conditions associated with posttraumatic stress disorder in U.S. older adults: results from wave 2 of the National Epidemiologic Survey on Alcohol and Related Conditions. J Am Geriatr Soc. 2012;60:296-303.

5. Schlenger WE, Corry NH, Williams CS, et al. A prospective study of mortality and trauma-related risk factors among a nationally representative sample of Vietnam veterans. Am J Epidemiol. 2015;182:980-90.

6. Pietrzak RH, Harpaz-Rotem I, Southwick SM. Cognitivebehavioral coping strategies associated with combat-related PTSD in treatment-seeking OEF-OIF Veterans. Psychiatry Res. 2011;189:251-8.

7. Pietrzak RH, Van Ness PH, Fried TR, Galea S, Norris F. Diagnostic utility and factor structure of the PTSD Checklist in older adults. Int Psychogeriatr. 2012;24:1684-96.

8. Schlenger WE, Mulvaney-Day N, Williams CS, et al. PTSD and use of outpatient general medical services among veterans of the Vietnam war. Psychiatr Serv. 2016;67:543-50.

9. Santiago PN, Ursano RJ, Gray CL, et al. A systematic review of PTSD prevalence and trajectories in DSM-5 defined trauma 
exposed populations: intentional and non-intentional traumatic events. PLoS One. 2013;8:e59236.

10. Broekman BF, Olff M, Boer F. The genetic background to PTSD. Neurosci Biobehav Rev. 2007;31:348-62.

11. Thakur GS, Daigle BJ Jr., Dean KR, et al. Systems biology approach to understanding post-traumatic stress disorder. Mol Biosyst. 2015;11:980-93.

12. Stein MB, Chen CY, Ursano RJ, et al. Genome-wide association studies of posttraumatic stress disorder in 2 cohorts of US army soldiers. JAMA Psychiatry. 2016;73:695-704.

13. Ehlers CL, Gizer IR, Gilder DA, Yehuda R. Lifetime history of traumatic events in an American Indian community sample: heritability and relation to substance dependence, affective disorder, conduct disorder and PTSD. J Psychiatr Res. 2013;47:155-61.

14. Stein MB, Jang KL, Taylor S, Vernon PA, Livesley WJ. Genetic and environmental influences on trauma exposure and posttraumatic stress disorder symptoms: a twin study. Am J Psychiatry. 2002;159:1675-81.

15. Manolio TA, Collins FS, Cox NJ, et al. Finding the missing heritability of complex diseases. Nature. 2009;461:747-53.

16. True WR, Rice J, Eisen SA, et al. A twin study of genetic and environmental contributions to liability for posttraumatic stress symptoms. Arch Gen Psychiatry. 1993;50:257-64.

17. Sartor CE, Grant JD, Lynskey MT, et al. Common heritable contributions to low-risk trauma, high-risk trauma, posttraumatic stress disorder, and major depression. Arch Gen Psychiatry. 2012;69:293-9.

18. Zannas AS, Provencal N, Binder EB. Epigenetics of posttraumatic stress disorder: current evidence, challenges, and future directions. Biol Psychiatry. 2015;78:327-35.

19. Klengel T, Pape J, Binder EB, Mehta D. The role of DNA methylation in stress-related psychiatric disorders. Neuropharmacology. 2014;80:115-32.

20. Smith AK, Conneely KN, Kilaru V, et al. Differential immune system DNA methylation and cytokine regulation in posttraumatic stress disorder. Am J Med Genet B Neuropsychiatr Genet. 2011;156B:700-8.

21. Uddin M, Aiello AE, Wildman DE, et al. Epigenetic and immune function profiles associated with posttraumatic stress disorder. Proc Natl Acad Sci USA. 2010;107:9470-5.

22. Van Gestel S, Houwing-Duistermaat JJ, Adolfsson R, van Duijn $\mathrm{CM}$, Van Broeckhoven C. Power of selective genotyping in genetic association analyses of quantitative traits. Behav Genet. 2000;30:141-6.

23. Chen YA, Lemire $M$, Choufani $S$, et al. Discovery of cross-reactive probes and polymorphic $\mathrm{CpGs}$ in the Illumina Infinium HumanMethylation450 microarray. Epigenetics. 2013;8:203-9.

24. Koch SB, van Zuiden M, Nawijn L, Frijling JL, Veltman DJ, Olff M. Intranasal oxytocin administration dampens amygdala reactivity towards emotional faces in male and female PTSD patients. Neuropsychopharmacology. 2016;41:1495-504.

25. Blake DD, Weathers FW, Nagy LM, et al. The development of a Clinician-Administered PTSD Scale. J Trauma Stress. 1995;8:75-90.

26. Sheehan DV, Lecrubier $\mathrm{Y}$, Sheehan $\mathrm{KH}$, et al. The MiniInternational Neuropsychiatric Interview (M.I.N.I.): the development and validation of a structured diagnostic psychiatric interview for DSM-IV and ICD-10. J Clin Psychiatry. 1998;59(Suppl 20):22-33. quiz34-57

27. First MBSR, Gibbon M, et al. Structured clinical interview for DSM-IV axis I disorders (SCID-I), clinician version, administration booklet. Washington D.C.: American Psychiatric Publishing Inc.; 2012.
28. Kamburov A, Stelzl U, Lehrach H, Herwig R. The ConsensusPathDB interaction database: 2013 update. Nucleic Acids Res. 2013;41:D793-800.

29. Untergasser A, Cutcutache I, Koressaar T, et al. Primer3-new capabilities and interfaces. Nucleic Acids Res. 2012;40:e115.

30. Tsai PC, Bell JT. Power and sample size estimation for epigenome-wide association scans to detect differential DNA methylation. Int J Epidemiol. 2015;44:1429-41.

31. Hoexter MQ, Fadel G, Felicio AC, et al. Higher striatal dopamine transporter density in PTSD: an in vivo SPECT study with [(99m) Tc]TRODAT-1. Psychopharmacology. 2012;224:337-45.

32. Michels KB, Binder AM, Dedeurwaerder S, et al. Recommendations for the design and analysis of epigenome-wide association studies. Nat Methods. 2013;10:949-55.

33. Jaffe AE, Murakami $\mathrm{P}$, Lee $\mathrm{H}$, et al. Bump hunting to identify differentially methylated regions in epigenetic epidemiology studies. Int J Epidemiol. 2012;41:200-9.

34. Friedman MJ, Wang S, Jalowiec JE, McHugo GJ, McDonaghCoyle A. Thyroid hormone alterations among women with posttraumatic stress disorder due to childhood sexual abuse. Biol Psychiatry. 2005;57:1186-92.

35. Lane JM, Liang J, Vlasac I, et al. Genome-wide association analyses of sleep disturbance traits identify new loci and highlight shared genetics with neuropsychiatric and metabolic traits. Nat Genet. 2017;49:274-81.

36. Siegmund KD, Connor CM, Campan M, et al. DNA methylation in the human cerebral cortex is dynamically regulated throughout the life span and involves differentiated neurons. PLoS One. 2007;2:e895.

37. Lavebratt C, Aberg E, Sjoholm LK, Forsell Y. Variations in FKBP5 and BDNF genes are suggestively associated with depression in a Swedish population-based cohort. J Affect Disord. 2010;125:249-55.

38. Cross-Disorder Group of the Psychiatric Genomics C. Identification of risk loci with shared effects on five major psychiatric disorders: a genome-wide analysis. Lancet. 2013;381:1371-9.

39. Sumner JA, Sheridan MA, Drury SS, et al. Variation in CACNA1C is associated with amygdala structure and function in adolescents. J Child Adolesc Psychopharmacol. 2015;25:701-10.

40. Dunn EC, Solovieff N, Lowe SR, et al. Interaction between genetic variants and exposure to Hurricane Katrina on posttraumatic stress and post-traumatic growth: a prospective analysis of low income adults. J Affect Disord. 2014;152-4:243-9.

41. van Zuiden M, Geuze E, Willemen HL, et al. Glucocorticoid receptor pathway components predict posttraumatic stress disorder symptom development: a prospective study. Biol Psychiatry. 2012;71:309-16.

42. Rice L, Waters CE, Eccles J, et al. Identification and functional analysis of SKA2 interaction with the glucocorticoid receptor. J Endocrinol. 2008;198:499-509.

43. Boks MP, Rutten BP, Geuze E, et al. SKA2 methylation is involved in cortisol stress reactivity and predicts the development of post-traumatic stress disorder (PTSD) after military deployment. Neuropsychopharmacology. 2016;41:1350-6.

44. Sadeh N, Spielberg JM, Logue MW, et al. SKA2 methylation is associated with decreased prefrontal cortical thickness and greater PTSD severity among trauma-exposed veterans. Mol Psychiatry. 2016;21:357-63.

45. Pandey GN, Rizavi HS, Zhang H, Bhaumik R, Ren X. The expression of the suicide-associated gene SKA2 is decreased in the prefrontal cortex of suicide victims but not of nonsuicidal patients. Int J Neuropsychopharmacol. 2016;19:pii: pyw015.

46. Guintivano J, Brown T, Newcomer A, et al. Identification and replication of a combined epigenetic and genetic biomarker predicting suicide and suicidal behaviors. Am J Psychiatry. 2014;171:1287-96. 
47. Kaut O, Schmitt I, Hofmann A, et al. Aberrant NMDA receptor DNA methylation detected by epigenome-wide analysis of hippocampus and prefrontal cortex in major depression. Eur Arch Psychiatry Clin Neurosci. 2015;265: 331-41.

48. Sakai M, Watanabe Y, Someya T, et al. Assessment of copy number variations in the brain genome of schizophrenia patients. Mol Cytogenet. 2015;8:46.

49. Connolly JJ, Glessner JT, Hakonarson H. A genome-wide .association study of autism incorporating autism diagnostic interview- revised, autism diagnostic observation schedule, and social responsiveness scale. Child Dev. 2013;84:17-33.

50. Bagot RC, Cates HM, Purushothaman I, et al. Circuit-wide transcriptional profiling reveals brain region-specific gene networks regulating depression susceptibility. Neuron. 2016;90:969-83.

51. Kolassa IT, Kolassa S, Ertl V, Papassotiropoulos A, De Quervain DJ. The risk of posttraumatic stress disorder after trauma depends on traumatic load and the catechol-o-methyltransferase Val(158) Met polymorphism. Biol Psychiatry. 2010;67:304-8.

\section{Affiliations}

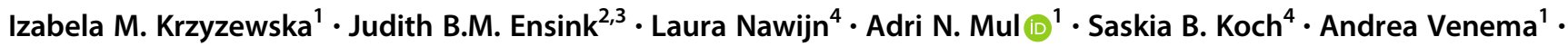
Vinod Shankar ${ }^{1}$ - Jessie L. Frijling ${ }^{4}$ - Dirk J. Veltman ${ }^{5} \cdot$ Ramon J.L. Lindauer $^{2,3} \cdot$ Miranda Olff $^{4,6} \cdot$ Marcel M.A.M. Mannens ${ }^{1} \cdot$ Mirjam van Zuiden $^{4} \cdot$ Peter Henneman $^{1}$

1 Department of Clinical Genetics, Genome Diagnostics laboratory, Academic Medical Center Amsterdam, Amsterdam, The Netherlands

2 Department of Child and Adolescent Psychiatry, Academic Medical Center, Amsterdam, The Netherlands

3 De Bascule, Academic Centre for Child and Adolescent Psychiatry, Amsterdam, The Netherlands
4 Department of Psychiatry, Anxiety Disorders, Academic Medical Center, Amsterdam, The Netherlands

5 Department of Psychiatry, VU University Medical Center, Amsterdam, The Netherlands

6 Arq Psychotrauma Expert Group, Diemen, The Netherlands 